\title{
Effect of age on visual acuity after cataract extraction
}

\author{
J L JAY,' R B MAMMO,' AND D ALLAN ${ }^{12}$ \\ From the ${ }^{1}$ Tennent Institute of Ophthalmology, Western Infirmary, Glasgow G11 6NT, and ${ }^{2}$ West of Scotland \\ Health Board's Department of Clinical Physics and Bio-Engineering, Glasgow
}

SUMmARY The best Snellen visual acuity in the three months after cataract extraction was found to decline with the age of the patient. The relationship was identical in 111 patients who had extracapsular extraction and a Pearce tripod posterior chamber intraocular lens and in 50 patients who had intracapsular extraction with spectacle correction. Vision after operation varied from a mean value of $6 / 5$ at 50 years to $6 / 12$ at 90 years, a decline of 1 line per 13.4 years. In the intracapsular group, over a mean follow-up period of 14 years, the rate of fall in acuity with increasing age after operation was found to be statistically similar to that of the early postoperative acuity plotted against age for both types of operation. This suggests that the rate of decline with age is unchanged after a prolonged period of aphakia with presumed increased exposure to ultraviolet and blue light. The data were found to be similar to the decline in the neurosensory elements of vision with age measured experimentally in 20 phakic subjects by laser interferometry. This method of assessment of contrast sensitivity threshold effectively bypasses changes in the optical media. The findings indicate that the previously recognised drop in visual acuity with age is not related to changes in the aging crystalline lens and support the view that there is a decline in the neurosensory elements of vision. It is important to recognise this deterioration so that results of surgery or other treatment are adjusted to allow for the age of the patients.

The reduction in visual acuity in the healthy human eye after the age of 45 years was reviewed by Weale in 1975.' The same aging phenomenon may be detected by measuring contrast sensitivity function at high spatial frequencies. ${ }^{23}$ Senile miosis seems to have too small an effect on retinal illumination to make any real contribution to the observed reduction in vision. Increased optical density and light scattering in the aging crystalline lens may be important, though a model designed to simulate cataract suggests this may not be a major factor, ${ }^{4}$ and Weale ${ }^{5}$ could not demonstrate any loss of transparency in human postmortem lenses with age.

Neurosensory changes with advancing years are probably more important. Morrison and $\mathrm{McGrath}^{6}$ have used laser interferometry to assess the 'nonoptical' components of vision in normal subjects. This method provides retinal stimulation independent of changes in the optical media, and the results suggest that deterioration with age is a function of changes in the neurosensory elements of vision. Indeed, significant neuron loss in the aging human

Correspondence to Dr J L Jay, Tennent Institute of Ophthalmology, Western Infirmary, Church Street, Glasgow G11 6NT. visual cortex has been confirmed by Devaney and Johnson. ${ }^{7}$

Our study examines the age related changes in visual acuity after surgical removal of the crystalline lens. The aim is not only to verify that the aging lens is not an important factor in the reduction of vision with increasing age but also to establish a reference base line of visual acuity over the age of 50 years. At present publications quoting visual results of surgery or other treatments make no allowance for age and may therefore be neglecting an important variable.

\section{Patients and methods}

Two groups of patients were chosen:

Group $A$ comprised one eye of each of 111 patients who had had uncomplicated extracapsular lens extraction with implantation of a Rayner-Pearce tripod posterior chamber lens. The operations were performed with the aid of an operating microscope with coaxial illumination. Patients with other ocular or systemic diagnoses which might have affected the visual acuity were excluded. Macular degeneration, amblyopia, diabetes, and corneal or vitreous 
opacities were the most common reason for exclusion. All cases had intact posterior capsules and none subsequently required secondary capsulotomy. In this series the rate of posterior capsulotomy has been less than $0.5 \%$ at four years. The mean age of the group was 68 years, range 41 to 89 years.

Group B consisted of one eye of each of 50 patients who had undergone uncomplicated intracapsular cataract extraction between 10 and 23 years previously (mean 14 years). The operations were performed without an operating microscope, and optical correction was by spectacle lenses. Cases excluded from the study were as listed for group A. In group B the mean age at operation was 62 years and the range 51 to 70 years. The mean age at final follow-up was 76 years, range 71 to 89 years.

The relationship between age at operation and the best Snellen visual acuity recorded in the three months following operation was analysed in both groups. In group B the visual acuity and age at final follow-up was also recorded, so that for each patient the final acuity could be compared with the acuity in the early postoperative period.

In a further analysis the slope of reduction in Snellen acuity with age as obtained above in the pseudophakic and aphakic groups was compared with the slope of the 'non-optical' and presumed neurosensory reduction of vision as measured by contrast sensitivity threshold using laser interferometry in healthy phakic eyes (data reworked from Morrison and $\mathrm{McGrath}^{6}$ ). These experimental contrast sensitivity data were measured at $40 \%$ contrast, whereas the clinical Snellen test type presents an uncertain contrast, probably in the region of $70 \%$. To make the two sets of data more comparable it was therefore necessary to adjust the comparative scale of cycles/degree to Snellen acuity so that the mean values of the two groups coincided. The age profile of the experimental group was similar to that in the clinical groups A and B (mean 67 years, range 53 to 86 years).

To facilitate the statistical analyses Snellen acuities were transformed according to a simple linear code with a unit inverval of one half of a Snellen line and increasing with improving acuity; an acuity of $6 / 18$ became 1 unit, 6/12 became 3, 6/9 became 5 units and so on. Linear regressions were performed to describe the variation of acuity code with age, and all regression line equations given in the text are expressed in terms of these transformed acuity codes. Product moment correlation coefficients were also calculated. The slopes of regression lines were compared by evaluating the appropriate $t$ statistic from the regression data. A two-tailed sign test, a paired, nonparametric procedure, was used to examine the changes in acuity of group B over their follow-up period. These calculations were performed with the aid of the MINITAB package on the University of Glasgow ICL 2988 computer system.

\section{Results}

Fig. 1 displays the age related reduction of Snellen acuity (mean \pm 1 SD) measured in the early postoperative period. Visual acuity dropped from about $6 / 5$ at 50 years to $6 / 12$ at 90 years. The relationship of age to visual acuity was statistically significant for both groups at $p<0.001$. The regression line for the extracapsular pseudophakia results, group A (acuity $=15.1-0.145 \times$ age; correlation coefficient $=-0.740$; $\mathrm{DF}=109)$ was not significantly different $(0 \cdot 1<\mathrm{p}<0 \cdot 2)$ from that of the intracapsular spectacle aphakia patients, group B (acuity $=19 \cdot 0-0 \cdot 200 \times$ age; correlation coefficient $=-0.638 ; \quad D F=48)$. A similar analysis could not detect any significant difference between males and females for either group $(0 \cdot 1<\mathrm{p}<0 \cdot 2)$.

Examination of the data on change in visual acuity within group B over the follow-up period (mean 14 years) showed the same gradient of deterioration with advancing years (Fig. 2). While there was a significant $(p<0.001)$ drop in acuity over the followup period, the regression line for the early postoperative results (acuity $=19 \cdot 0-0.200 \times$ age, as above) was not significantly different $(0 \cdot 1<\mathrm{p}<0 \cdot 2)$ from that of the visual acuity at final follow-up (acuity $=12.7-$ $0 \cdot 106 \times$ age; correlation coefficient $=-0.267 ; \mathrm{DF}=$ 48). Males did not differ from females in this analysis $(0 \cdot 2<\mathrm{p}<0 \cdot 5)$. There was, however, a much wider scatter of the data in the follow-up recordings, reflected in the poorer correlation coefficient. We

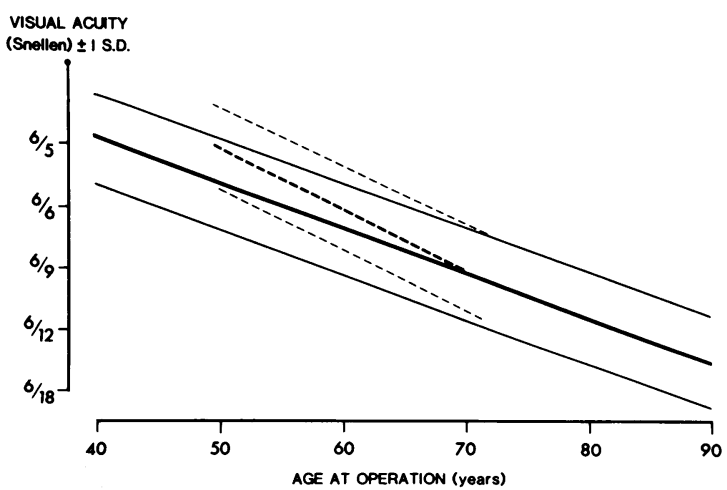

Fig. 1 Regression lines ( \pm 1 standard deviation) showing the decline with age of best Snellen visual acuity measured in the first three months after cataract extraction. Solid lines represent extracapsular surgery with intraocular lens and dotted lines intracapsular surgery with spectacle correction. The two groups are not statistically different. 


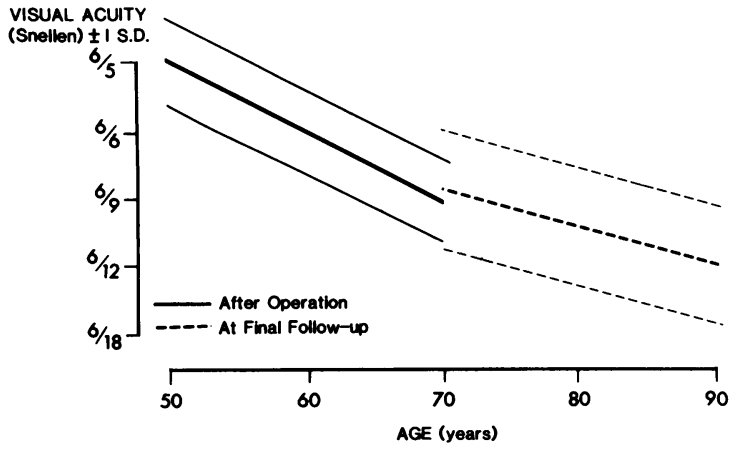

Fig. 2 Visual acuity of group B in the three months after operation (solid lines) and after average follow up of 14 years (dotted lines) plotted against age. Statistical analysis suggests that the rate of decline with age is not altered by the period of aphakia.

therefore sought to verify the observation by comparing the slope of visual acuity at final follow-up in group B (age range 71 to 89 years) with an age matched population selected from group $A$. This confirmed that there was no significant difference $(0 \cdot 2<\mathrm{p}<0 \cdot 5)$.

In Fig. 3 the slope of threshold frequency for laser interference fringes against age in the experimental study of 20 subjects is compared with the slope of postoperative visual acuity against age at operation in groups A and B combined. The reduction in spatial frequency discrimination in the experimental group showed a trend which was similar to that for Snellen acuity in the clinical groups. However, they were not identical. Statistical analysis showed the slopes to be significantly different $(0 \cdot 01<\mathrm{p}<0 \cdot 02)$.

\section{Discussion}

The results indicate that the best corrected Snellen visual acuity after cataract extraction declines with

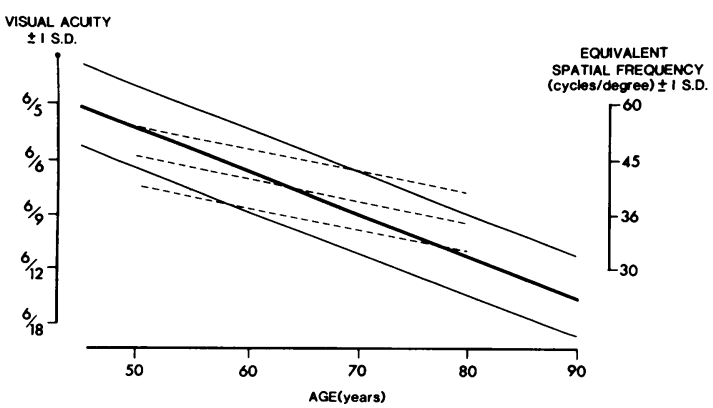

Fig. 3 Combined data of groups $A$ and $B$ (solid lines) show a similar reduction of Snellen visual acuity with age to the reduction of threshold frequency for laser interference fringes with age (dotted lines), from Morrison and McGrath. ${ }^{6}$ the age of the patient by 1 line per 13.4 years from $6 / 5$ at 50 years to $6 / 12$ at 90 years. Thus one would expect an average acuity of $6 / 6$ at 63 years and $6 / 9$ at 77 years. The relationship of visual acuity to age is identical in contemporary extracapsular extractions with posterior chamber intraocular lenses and in retrospective observations in intracapsular operations with spectacle correction. These different surgical techniques might have age related subclinical complications causing minor changes in acuity, but, as the two main potential complications, capsule opacification in extracapsular operations and macular oedema in intracapsular operations, are confined to each individual group, it is unlikely they would influence the results to an identical degree. In the analyses of visual acuity in the early postoperative period (Fig. 1) we assume that the decline with age has occurred before operation. This figure is very similar to Weale's display ${ }^{8}$ of the slope of decline of visual acuity as a function of age in healthy phakic subjects. It also confirms the suggestion made by Owsley et al. ${ }^{9}$ that for tests of contrast sensitivity at the higher spatial frequencies up to 18.2 cycles/degree there is no difference between older healthy phakic eyes and those in which an acrylic intraocular lens has replaced the crystalline lens.

It therefore appears that the deterioration in vision is not related to changes in the crystalline lens. Furthermore the same relationship is confirmed when the change in visual acuity is examined after a long period of aphakia (Fig. 2), so that, in the absence of identifiable disease, the condition of aphakia neither accelerates nor retards the deterioration of visual acuity with advancing years. This is an interesting observation in view of the current interest in phototoxic retinal effects of ultraviolet and blue light. ${ }^{10-13}$

We have treated the data on the assumption that the trend is part of a linear function, and this may be a crude though justifiable approximation. The wider scatter of data in the follow-up observations in Fig. 2 may conceal an alteration in the gradient of reduction in vision in the very old patients. We would also expect the slope to be flatter towards the younger end, as previous data ${ }^{1}$ suggest that only over 45 years of age is there a fall off in visual acuity. We have insufficient data at the extreme ends of the age range to examine these possibilities.

Our findings in pseudophakic and aphakic patients are similar to those of the experimental assessment of non-optical components of vision in the study by Morrison and McGarth. ${ }^{6}$ This again suggests that the reduction in vision with advancing years is not a function of changes in the crystalline lens but a result of degenerative changes in the neurosensory components of vision. The lines shown in Fig. 3 are not 
identical, but they represent radically different test systems with different effects on wide angle light scatter and chromatic aberration. Therefore the slightly different gradients could still represent the same phenomenon. In addition the small number of observations in the laser interference group limits the scope for statistical comparison.

Whatever the theoretical considerations, it is important in assessing clinical results after cataract surgery or indeed after any form of therapy, that the visual acuity is adjusted to allow for the decline in visual acuity with age.

We thank Dr James Morrison for his helpful advice and encouragement, and Mrs Jennifer Murray for typing the manuscript.

\section{References}

1 Weale RA. Senile changes in visual acuity. Trans Ophthalmol Soc UK 1975; 95: 36-8.

2 McGrath C, Morrison JD. The effects of age on spatial frequency perception in human subjects. $Q J$ Exp Physiol 1981; 66: 253-61.

3 Ross JE, Clarke DD, Bron AJ. Effect of age on contrast sensitivity function: uniocular and binocular findings. $\mathrm{Br} J$ Ophthalmol 1985; 69: 51-6.

4 Zuckerman JL, Miller D, Dyes W, Keller M. Degradation of vision through a simulated cataract. Invest Ophthalmol Vis Sci 1973; 12: 213-24.

5 Weale RA. Transparency and power of post-mortem human lenses: variation with age and sex. Exp Eye Res 1983; 36: $731-41$.

6 Morrison JD, McGrath C. Assessment of the optical contributions to the age related deterioration in vision. $Q J$ Exp Physiol 1985; 70: 249-69.

7 Devaney KO, Johnson HA. Neuron loss in the ageing visual cortex of man. J Gerontol 1980; 35: 836-41.

8 Weale RA. A biography of the eye. London: Lewis, 1982: 279.

9 Owsley C, Gardner T, Sekuler R, Lieberman H. Role of the crystalline lens in the spatial vision loss in the elderly. Invest Ophthalmol Vis Sci 1985; 26: 1165-70.

10 Sperduto RD, Hiller R, Seigel D. Lens opacities and senile maculopathy. Arch Ophthalmol 1981; 99: 1004-8.

$11 \mathrm{McDonald}$ RH, Irvine AR. Light induced maculopathy from the operating microscope in extracapsular cataract extraction and intraocular lens implantation. Ophthalmology (Rochester) 1983; 90: 945-51.

12 Berler DK, Peyser R. Light intensity and visual acuity following cataract surgery. Ophthalmology (Rochester) 1983, 90: 933-6.

13 Ross WH. Light induced maculopathy. Am J Ophthalmol 1984; 98: 488-93. 